\title{
Controlling hilft!
}

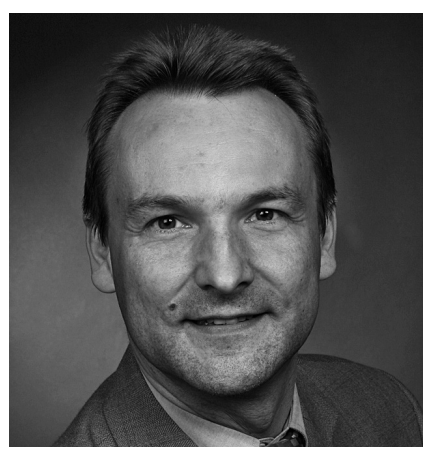

VON MARTIN KELTER

Martin Kelter ist Dozent an der

Hochschule Heilbronn, Campus

Künzelsau - Reinhold-Würth-

Hochschule im Studiengang

Sozialmanagement mit den

Schwerpunkten operatives Con-

trolling in Sozialbetrieben und

Vereinsmanagement. Zuvor war

er langjähriger Leiter Controlling

und Abteilungsleiter Verwaltung

in einer Sozialorganisation.

martin.kelter@hs-heilbronn.de

\author{
Vor allem kleine soziale Organisationen und \\ Unternehmen verzichten aus personellen und \\ wirtschaftlichen Gründen auf ein umfassendes \\ Controlling-Konzept - und provozieren auf \\ diese Weise fatale Managementfehler. \\ Dabei können bereits einfach Instrumente helfen, \\ Fehlentwicklung rechtzeitig zu erkennen.
}

Durch die Gesetzesreformen der letzten Jahrzehnte sowie die Kürzungen staatlicher Zuschüsse ist es für soziale Organisationen und Unternehmen unabdingbar geworden, effizient $\mathrm{zu}$ wirtschaften und die Mittel rational einzusetzen. Sie müssen sich - auch organisatorisch - den veränderten Rahmenbedingungen anpassen und sind gezwungen, Strategien und Maßnahmen zu entwickeln, um diesen Veränderungen zu begegnen.

In der Realität arbeiten bedarfswirtschaftliche Unternehmungen zu selten mit Kennzahlen oder Strategien. Strategische Planung findet nur - oft spontan - in Einzelgesprächen zwischen Vorstandsmitgliedern und der Geschäftsführung statt. Ein schlüssiges System, das Strategien in operative Zielvereinbarungen umsetzt und die handelnden Mitarbeiterinnen und Mitarbeiter einbezieht, existiert selten. Die operative Planung besteht oft aus fortgeschriebenen Vorjahresbudgets. Eine Überprüfung auf Machbarkeit und Zieladäquanz findet nicht oder kaum statt.

Die Mitarbeitenden, die die Umsetzung der geplanten Maßnahmen vornehmen sollen, erhalten nur unzureichende Budget- und Kostenstellenvorgaben. Informationen über die unterjährigen Ist-Daten erhalten sie nicht oder wenig. Eine ständige Überprüfung der Zahlen findet nicht statt und ist auch aufgrund der schlechten operativen Planungsvorgaben eher sinnlos.
Hieraus können sich wirtschaftliche Krisen einer Sozialorganisation entwickeln, die von den Beteiligten nicht erkannt oder verborgen werden - in beiden Fällen droht die Zahlungsunfähigkeit. Ein auf die Bedürfnisse in der Sozialwirtschaft angepasstes Controllingsystem ist notwendig, existiert aber oft nicht!

\section{Vier operative Instrumente der Gegensteuerung}

Es ist sicherlich aus wirtschaftlichen Gründen nicht vertretbar, für jede Sozialorganisation ein vollständiges (und somit teures) Controllingsystem einzuführen. Die folgende Darstellung praxiserprobter Instrumente soll daher ein durchführbares und aufeinander aufbauendes System von Controllinginstrumenten vorstellen, das jede Sozialorganisation einführen kann. Aufgrund des begrenzten Platzangebotes wird in diesem Artikel auf die Darstellung der strategischen Instrumente verzichtet. Es werden dafür die (bei einer wirtschaftlichen Schieflage zuerst einzusetzenden) kurz- und mittelfristigen Instrumente dargestellt.

\section{Erstes Instrument: der Liquiditätsplan}

Der Liquiditätsplan bildet die Basis des wirtschaftlichen Überlebens einer Sozialorganisation. Der Liquiditätsplan soll- 
te die nächsten zwölf Monate umfassen, wobei der erste Monat in Wochen unterteilt sein sollte, und für jede Position eine monatliche Planzahl enthalten. Dies ist für das Erkennen der mittelfristigen Überlebensfähigkeit sinnvoll und notwendig.

Auf Basis der aus der Finanzbuchhaltung zu erhaltenen Summen- und Saldenliste werden die liquiditätswirksamen Zahlungspositionen herausgefiltert und durch auf Vergangenheitsverläufen aufbauenden Zahlungsinformationen ein Liquiditätsplan erstellt.

Die von den Vergangenheitsverläufen und Vergangenheitswerten abweichenden Positionen müssen mit den ehren- und hauptamtlichen (Kostenstellen-) Verantwortlichen erarbeitet werden. Es ist davor zu warnen, dies ohne die Mitarbeitenden, die unmittelbare Kenntnisse des Marktes haben, zu erstellen.

Aus Rationalitätsgesichtspunkten ist es wichtig, sich auf die großen und bedeutsamen Positionen zu konzentrieren und die kleinen (z. B. Büromaterial) aus der Vergangenheit zu übernehmen. Eine regelmäßige, je nach wirtschaft-

\section{Die Betriebswirtschaftliche Auswertung (BWA)}

basiert auf den laufenden Daten der Finanzbuchhaltung. Sie gibt dem Unternehmer während des laufenden Finanzjahres Auskunft über seine Kosten- und Erlössituation und damit über die Ertragslage. Im Gegensatz zur Bilanz, die meist erst mit einigen Monaten Zeitverzögerung erstellt wird, kann die Betriebswirtschaftliche Auswertung die Zahlen zur aktuellen Lage des Unternehmens liefern. Die erste Betriebswirtschaftliche Auswertung wurde Ende der $1960 e$ Jahre von der DATEV in Deutschland als sogenannte DATEV-Standard-BWA Nr. 1 eingeführt. Sie dient oft als Entscheidungsgrundlage für kleine und mittelständische Unternehmen. Für Kreditinstitute ist sie das wesentliche Informationsinstrument für die Beurteilung von Krediten gem. § 18 KWG im Rahmen der Kreditwürdigkeitsprüfung. Mittlerweile hat die DATEV als Trendsetter um die Standard-BWA herum eine erhebliche Vielfalt von zusätzlichen Auswertungen geschaffen (Controllingreport, Kapitalflussrechnung, branchenbezogene BWA für Steuerberater, Rechtsanwälte, Optiker, Kfz-Handel u. v. a. m.). Diese DATEV-BWA wurden von anderen Rechnungswesen-Anbietern eins zu eins übernommen.

Quelle: www.wikipedia.de

die Ist-Erlöse oder die Planerlöse dargestellt.

Das Ziel dieser Analyse ist es herauszufinden, welche Erlöse für die Sozialorganisation besonders wichtig und besonders anfällig für Rückgänge sind. Diesem liegt die Überlegung zugrunde, dass es Erlöse gibt, die sich schneller verringern, als auf der Gegenseite Kosten zur Kompensation verringert werden können.

\section{"In die Prüfung abweichender Positionen müssen die Mitarbeitenden einbezogen werden, da sie unmittelbare Kenntnisse des Marktes haben"}

licher Schieflage wöchentliche, monatliche, quartalsweise Überarbeitung des Liquiditätsplans ist erforderlich.

Fragt man nach den Gründen, warum ein relativ einfaches Instrument wie der Liquiditätsplan in vielen Sozialorganisationen fehlt, stellt man fest, dass entweder die Notwendigkeit nicht gesehen wird, das betriebswirtschaftliche Knowhow oder die quantitativen Kapazitäten fehlen.

\section{Zweites Instrument: die Risikostrukturanalyse}

Die Risikostrukturanalyse der Erlöse erlaubt wertvolle Erkenntnis für die kurzfristige Strategie einer sozialen Organisation. Sie entsteht gleichsam als Nebenprodukt der Liquiditätsplanung. In Form eines Kreisdiagramms werden
Das Augenmerk des Managements muss also auf diesen risikoreichen und bedeutsamen Erlösen liegen. So ist beispielsweise bei einem Fall des negativen Images (z. B. durch Presseartikel über mögliche wirtschaftliche Schieflagen) in der Öffentlichkeit über gezielte öffentlichkeitswirksame Aktionen nachzudenken.

\section{Drittes Instrument: die monatliche Soll-Ist-Abweichungsanalyse}

Die Soll-Ist-Abweichungsanalyse entsteht im Rahmen von Besprechungen mit den Kostenstellenverantwortlichen. Dieses Instrument ist das Kernstück des hier vorgestellten Instrumentariums. Es werden Planung, Kontrolle und Steuerung in Zusammenarbeit mit den verantwortlichen Mitarbeiterinnen und
Mitarbeitern (Kostenstellenverantwortlichen) erstellt und ausgewertet.

Voraussetzung für dieses Instrument ist eine Planung, die auf Monatswerten basiert. Für jede Kostenstelle (mindestens aber für jeden Bereich) muss eine Planung auf Zeilenebene der betriebswirtschaftlichen Auswertung (BWA, vgl. Kasten), besser auf Kostenartenebene, erstellt werden.

Hinsichtlich der monatlichen Planwerte ist es auch hier wieder sinnvoll, auf die Erfahrungen der Liquiditätsplanung und der Vorjahre zurückzugreifen. Es ist davon abzuraten, einen Jahresplanwert der Einfachheit halber linear auf die Monate zu verteilen, da es hierbei zu falschen Steuerungsinformationen kommen kann. Aus Vereinfachungsgründen sind nur die wichtigen BWA-Zeilen und BWA-Konten mit den erwarteten Monatsschwankungen zu planen und die restlichen Zeilen und Konten linear zu planen. So wie die Planung mit den Bereichs- und Kostenstellenverantwortlichen durchgeführt werden sollte, sind auch die monatlichen Plan-Ist-Auswertungen mit den entsprechenden Verantwortlichen mittels Kostenstellenleiterbesprechungen durchzuführen.

In diesen Besprechungen werden die Ist-Zahlen analysiert, mit den PlanZahlen verglichen und Gründe für die Abweichungen dargestellt. Es wird hierbei automatisch dazu kommen, dass die möglichen Reaktionen (zukünftigen Aktionen) in diesem Geschäftsfeld besprochen werden. Ein Einbezug und Identifikation der betroffenen Verantwortlichen ist die Folge. 


\section{Viertes Instrument: der monatliche Vorstandsreport}

Der monatliche Vorstandsreport bildet die zielgruppenorientierte Kurzinformation. Gerade bei einem ehrenamtlichen Vorstand mit dem Problem der begrenzten Zeitressourcen sowie dem begrenzten Einblick in das Tagesgeschehen - aber mit vollem Haftungsrisiko - ist es notwendig, die wichtigsten wirtschaftlichen Informationen vereinfacht auf einer Druckseite dem Vorstand in der (monatlichen) Vorstandssitzung zu präsentieren. Dies ist durch eine hohe Verdichtung der Zahlen (aber auf Bereichs- und Abteilungsebene und nicht auf Gesamtorganisationsebene) sowie die Nutzung von (Warn-) Farben und Pfeilen möglich. Dieses Vorgehen hat den Vorteil, dass in einer überschaubaren Zeitspanne der Vorstand informiert und in die (strategische) Diskussion eingebunden wird.

\section{Fazit und Ausblick}

Die Fehler im Management einer NonProfit-Organisation sind vielschichtig, letztendlich beruhen jedoch viele Fehler auf fehlenden oder zurückgehaltenen Informationen.

Im Kern geht es um die Einführung eines controllingorientierten Managements, das auf die Gegebenheiten der betreffenden Sozialorganisation angepasst werden muss - unter Beachtung der Größe, der Geschäftsfelder und der vorhandenen Ressourcen. Wichtig ist die Einbindung der Verantwortlichen sowie der freie Fluss und Zugang der für die Handelnden notwendigen (!) Informationen um die Sozialorganisationen weniger fehlerhaft und mehr zielorientiert zu gestalten.

\section{Literatur}

Horak, Christian (1995): Controlling in Nonprofit-Organisationen, 2. Auflage, Wiesbaden: Deutscher-Universitätsverlag.

Tiebel, Christoph (2006): Management in Non Profit Organisationen, München: Verlag Vahlen. Tiebel, Christoph/Kelter, Martin: Fehler im NPO-Management und Instrumente der Gegensteuerung, in: Schauer, Reinbert/Helmig, Bernd/Purtschert, Robert/Witt, Dieter (Hg.) (2008): Steuerung und Kontrolle in Nonprofit -Organisationen, Linz: Trauner Verlag.

\section{Alles in einem Band}

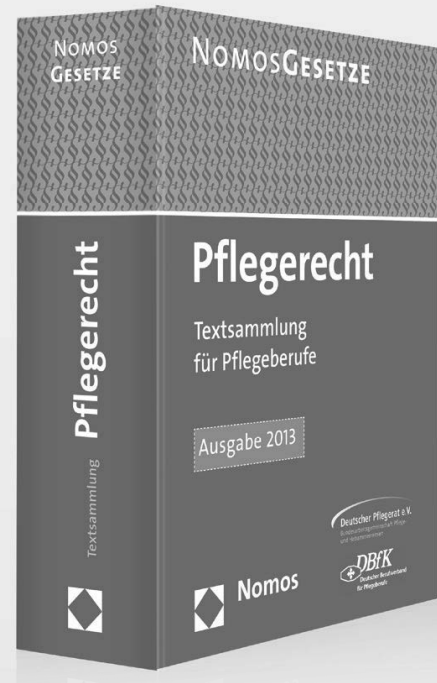

\section{Pflegerecht}

Textsammlung für Pflegeberufe

Ausgabe 2013

2013, 2.090 S., brosch., 24,- $€$ ISBN 978-3-8329-7963-8
Die Nomos Textsammlung „Pflegerecht“

bietet Ausbildung und Praxis der Pflegeberufe eine handliche Gesamtdarstellung aller wichtigen Rechtsgrundlagen in einem Band. Die praxisgerechte Auswahl der Vorschriften erfolgt in enger Kooperation mit dem Deutschen Berufsverband für Pflegeberufe und dem Deutschen Pflegerat.

Auf dem allerneuesten Stand des „Pflege-Neuausrichtungs-Gesetzes“ sind dabei auch die wichtigsten Länderregelungen berücksichtigt. Sie finden auf über 2.000 Seiten für nur 24,-- Euro alle relevanten Vorschriften, wie z.B.

- die Sozialgesetzbücher und das Pflegegesetz

- die Pflegegesetze der Länder, Pflege-Richtlinien und Pflegetransparenzvereinbarungen

- die Gesetze der Länder über den Öffentlichen Gesundheitsdienst

- alle Landesheimgesetze

- die Unterbringungs- bzw. Psychischkrankengesetze der Länder

- das Alten-, Krankenpflege-, Heilpraktiker- und Hebammengesetz sowie die zugehörigen Ausbildungs- und Prüfungsverordnungen

- die Arbeitsschutzgesetze (z.B. Arbeitszeitgesetz)

- das Vormünder- und Betreuungsvergütungsgesetz mit zugehörigen Mustern und Empfehlungen

Weitere Informationen: www.nomos-shop.de/19908

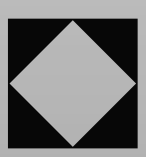

Nomos 